\title{
Research on Basic Public Cultural Services in Jiangxi Province of China: Current Situation, Problems and Countermeasures
}

\author{
Shaogang Liao*, Xiaomei Yu, Zhikang Luo \\ School of Finance and Public Administration, Jiangxi University of Finance and Economics, Nanchang, China
}

Email address:

liaoshaogang@163.com (Shaogang Liao)

*Corresponding author

\section{To cite this article:}

Shaogang Liao, Xiaomei Yu, Zhikang Luo. Research on Basic Public Cultural Services in Jiangxi Province of China: Current Situation, Problems and Countermeasures. International Journal of Economic Behavior and Organization. Vol. 8, No. 3, 2020, pp. 49-56. doi: $10.11648 /$ j.ijebo.20200803.11

Received: June 9, 2020; Accepted: June 28, 2020; Published: July 62020

\begin{abstract}
Basic public cultural services are based on the consensus of contemporary Chinese social development, and are provided by the government according to the current economic and social development stage and overall level, and are designed to protect citizens' basic cultural rights and interests. Basic public cultural services meet the basic cultural needs of the public and are therefore closely related to citizens' rights in cultural life. Especially under the goal of building a service-oriented government, in recent years China has continuously deepened its emphasis on the equalization of basic public cultural services provided by the government. Jiangxi Province, as an underdeveloped region in China, has obvious differences in basic public cultural services in different cities. The article combines the relevant statistical data of basic public cultural services in various cities in Jiangxi Province from 2016 to 2018 to calculate the equalization coefficient and coefficient of variation of basic public cultural services in Jiangxi Province, and measures the basic situation of 11 cities and measures between different cities The relative gap in the equalization of basic public cultural services. The research shows that the unequal characteristics of basic public cultural services among cities in Jiangxi Province are very obvious. In order to promote the rational allocation of resources in various cities in Jiangxi Province, narrow the gap between cities and cities, and achieve the equalization of high-quality and sustainable basic public cultural services, it is necessary to promote coordinated development between regions, establish a multi-input mechanism, and strengthen the construction of talents for basic public cultural services, improve the basic public cultural service infrastructure construction.
\end{abstract}

Keywords: Basic Public Cultural Services, Jiangxi Province, Equalization Coefficient, Coefficient of Variation

\section{Introduction}

With the increasing importance of the Chinese government to the cultural industry and the in-depth reform of China's market economy system, the Chinese cultural market has become increasingly prosperous, and the basic public cultural service industry has achieved rapid development. However, compared with the basic public cultural services in western developed countries, the regional development of China's basic public cultural services is not balanced. Basic public cultural services are better in the economically developed areas of Beijing and Shanghai, but lower in the economically underdeveloped areas. Promoting the development of basic public cultural services is an important way to activate the vitality of cultural industries in underdeveloped areas and improve the quality of life of residents. Basic public cultural services are cultural services that protect the basic cultural rights of the public and meet the basic cultural needs of the public. It provides a realistic reference basis for the coordinated improvement path of the basic public service supply level between regions and regions. Chinese and foreign scholars have studied the diverse objects of equalization of public cultural services from the perspectives of equalization of results, equalization of financial resources, equalization of rights and opportunities, and equalization of capabilities, the realization path of equalization of public cultural services. 
This article analyzes the impact of the uneven development of urban areas in Jiangxi on the equalization of basic public cultural services in Jiangxi, and guarantees the relatively undeveloped cities to enjoy basic public cultural services fairly, which has become an urgent theoretical and practical proposition.

\section{Basic Public Cultural Service Indicator System Construction and Calculation Method}

\subsection{The Meaning of Basic Public Cultural Services and Their Equalization}

Public culture is a culture that has obvious public characteristics and is shared by the general public, including both material and spiritual levels. It also shares cultural resources through public cultural places such as libraries and cultural art exhibition halls. Public cultural services refer to the public goods and services provided by the public sector and the quasi-public sector that satisfy the basic spiritual and cultural enjoyment of the public. The purpose is to protect the basic cultural rights of the public and enhance cultural soft power. Public culture can be understood from two dimensions. One is the connotation dimension, public culture is a sense of identification of people with cultural values, it emphasizes the cultivation of people's public concepts and a sense of belonging to social groups, while pursuing cultural integration and development harmonious society. The second is the extension dimension, which emphasizes that public culture is a culture with external public characteristics such as popularity and sharing. It takes public cultural places such as cultural art galleries and mass cultural station museums as carriers to provide people with participatory and shared culture with hierarchical characteristics.

The equalization of basic public cultural services can be understood from three aspects. One is the fairness of rights, the public has a fair right to enjoy basic public cultural services, its fairness means that all members of a country's society have the right to enjoy basic public cultural services, they are not treated unfairly because of differences in age, gender, region, and race, all societies Members can equally enjoy the public cultural products or cultural services provided by the state, and all have the right to enjoy basic public cultural services fairly. The second is that it has an essential foundation, the basics of public cultural services are embedded in the process, the basics emphasize a low-level and indispensable service, which is determined by the level of economic development of a country, as long as the low-level cultural needs are met It is possible to provide a higher level of service. Third, it is procedural, equalization of basic public cultural services is a constantly changing process, the types of public cultural services required by society and the ability to provide public cultural services are advancing and rising with the degree of social and economic development, it takes some time to realize equalization of cultural services.

\subsection{Construction of the Index System of Basic Public Cultural Services in Jiangxi Province}

To build a basic public cultural service indicator system, it is necessary to follow the prerequisites of scientific feasibility and other aspects. Based on the above definition of the basic public cultural service connotation, this paper builds two-level dimension system. In the input dimension, set a secondary indicator of per capita cultural undertakings; In the output dimension, eight secondary indicators are set: the number of libraries per 10000 people, the number of cultural centers per 10000 people, the number of museums per 10000 people, the collection of books in public libraries per capita, the comprehensive population coverage of broadcasting, and the comprehensive population coverage of television, the number of art performance groups per 10000 people, the number of cultural undertakings per 10000 people. And use the expert scoring method to determine the corresponding index weight, as shown in Table 1.

Table 1. Basic public cultural service index design and weight setting.

\begin{tabular}{llll}
\hline First-level indicators & Numbering & Secondary indicators & Weight coefficient \\
\hline Public cultural input & X1 & Cultural expenses per capita & 0.182 \\
& X2 & the number of libraries per 10000 people & 0.095 \\
& X3 & the number of museums per 10000 people & 0.075 \\
& X4 & the number of cultural centers per 10000 people & 0.085 \\
Xublic cultural output & X5 & Per capita possession of books in public libraries & 0.085 \\
& X6 & Broadcast comprehensive population coverage & 0.065 \\
& X7 & TV comprehensive population coverage & 0.088 \\
& X8 & the number of cultural undertakings per 10000 people & 0.165 \\
& X9 & the number of art performance groups per 10000 people & 0.078 \\
\hline
\end{tabular}

\subsection{Coefficient of Variation of Basic Public Cultural Services}

The coefficient of variation is the ratio of the standard deviation to the average, which reflects the degree of variation of a group of data. The formula is $\mathrm{Yi}=\mathrm{S} / \mathrm{X}$. In the formula, $\mathrm{Y}$ represents the coefficient of variation of the $\mathrm{i}$-th index, $\mathrm{S}$ is the standard deviation of the selected index, and $\mathrm{X}$ is the average of the selected index. When the coefficient of variation interval is less than 0.2 , the gap between the basic public cultural service supply is not obvious between the cities, 0.2-0.4 indicates that the gap is obvious, 0.4-0.6 indicates that the gap is large, and greater than 0.6 indicates that the gap is huge. It can be seen that the larger the value of Yi, the greater the fluctuation between the data, and the gap between the basic public cultural service levels of various cities is large. Otherwise, the gap between the basic public cultural service supply of various cities is small. 


\subsection{Equalization Coefficient of Basic Public Cultural Services}

The equalization coefficient is based on the variation index calculated above, and then the weight of each individual index is used to perform weighted summation to obtain the equalization coefficient. The function formula is: $\mathrm{Z}=\Sigma(\mathrm{Yi} \times \mathrm{Ti})$. In the formula, the coefficient of variation of an evaluation index of $\mathrm{Yi}$, $\mathrm{Ti}$ is the weight of an evaluation index, $\mathrm{Z}$ is the equalization coefficient obtained by adding the coefficients of variation, the larger the value, the greater the degree of gap between cities On the contrary, it means that the gap in the supply of basic public cultural services between cities is smaller.

\section{Current Situation of Basic Public Cultural Services in Jiangxi Province}

\subsection{Calculation of Variation Coefficient of Basic Public Cultural Services in Prefectures and cities In Jiangxi Province}

First of all, according to the "13th Five-Year Plan for Promoting Equalization of Basic Public Services", "Guidelines for National Basic Public Cultural Services (2015-2020)", "Opinions on Accelerating the Construction of a Modern Public Cultural Service System", Policy documents such as the "Five-Year Plan for the Promotion of Equalization of Basic Public Services" are used to select the index content.

Table 2. [1] 2018 Variation Coefficient of Basic Public Cultural Services in Jiangxi Province.

\begin{tabular}{|c|c|c|c|c|c|c|c|c|c|}
\hline Area & X1 & $\mathrm{X} 2$ & X3 & X4 & X5 & X6 & $\mathbf{X} 7$ & X8 & X9 \\
\hline Nanchang & 81.68 & 0.01 & 0.021 & 0.01 & 0.226 & 98.16 & 99.19 & 5.321 & 0.08 \\
\hline Jingdezhen & 31.03 & 0.006 & 0.015 & 0.006 & 0.118 & 100 & 100 & 1.569 & 0.002 \\
\hline Pingxiang & 8.82 & 0.006 & 0.003 & 0.006 & 0.103 & 99.59 & 99.95 & 1.51 & 0.009 \\
\hline Jiujiang & 63.88 & 0.015 & 0.018 & 0.015 & 0.351 & 97.7 & 98.73 & 1.327 & 0.027 \\
\hline Xinyu & 16.38 & 0.004 & 0.003 & 0.004 & 0.086 & 99.94 & 99.94 & 3.265 & 0.001 \\
\hline Yingtan & 2.52 & 0.004 & 0.005 & 0.004 & 0.05 & 96.96 & 97.59 & 2.174 & 0.013 \\
\hline Ganzhou & 49.65 & 0.019 & 0.018 & 0.019 & 0.446 & 95.97 & 97.83 & 3.758 & 0.027 \\
\hline Yichun & 24.82 & 0.011 & 0.012 & 0.011 & 0.157 & 99.17 & 98.4 & 2.335 & 0.018 \\
\hline Fuzhou & 37.47 & 0.014 & 0.01 & 0.014 & 0.165 & 99.43 & 99.76 & 3.016 & 0.086 \\
\hline Shangrao & 39.23 & 0.013 & 0.02 & 0.013 & 0.164 & 100 & 100 & 2.874 & 0.073 \\
\hline Average & 35.07 & 0.011 & 0.013 & 0.011 & 0.194 & 98.811 & 99.212 & 2.641 & 0.032 \\
\hline standard deviation & 22.321 & 0.005 & 0.006 & 0.005 & 0.115 & 1.341 & 0.888 & 1.129 & 0.031 \\
\hline coefficient of variation & 0.636 & 0.454 & 0.462 & 0.454 & 0.613 & 0.0136 & 0.009 & 0.427 & 0.968 \\
\hline
\end{tabular}

It can be seen from Table 2 that among the nine indicators of basic public cultural services in various cities in 2018, the indicators of Nanchang City and Ganzhou City are higher among 11 cities, Nanchang City is the capital city of Jiangxi Province, and is The provincial political, economic and cultural center, and Ganzhou City is the largest city in Jiangxi Province. Therefore, these two cities have obvious economic advantages, strong regional comprehensive strength, and almost all indicators of the index system are higher than other cities. Since Yingtan City, Xinyu City, and Pingxiang City rely on traditional resource-based industries to develop their economies, they are far from adapting to the development of today' s market economy. In addition, the basic public cultural services are less invested, so these three cities are basically developing. Compared with other cities, public cultural services are relatively backward. In the table, the coefficients of variation of the three indicators of per capita cultural undertakings, public library collections for ten thousand people, and art performance groups for ten thousand people are more than 0.6 , indicating a huge gap in public cultural services in these three areas. The coefficients of variation of the four indicators of the number of libraries owned by 10,000 people, the number of public museums owned by 10,000 people, the number of cultural museums owned by 10,000 people, and the number of employees employed by 10,000 people in cultural undertakings range from 0.4 to 0.6 , indicating these three aspects among 11 cities The public cultural service gap is relatively large. The equalization degree of radio and television coverage is the highest, both of which are less than 0.1 , indicating that the gap in radio and television coverage between cities is not obvious.

\subsection{Estimation of the Equalization Coefficient of Basic Public Cultural Services in Prefectures and Cities in Jiangxi Province}

This article can calculate the equalization coefficient from 2016 to 2018 based on the "Statistical Yearbook of Jiangxi Province" (2017-2019) and the statistical data of the New Tourism Bureau of the cities, cities, and regions in the past three years.

Table 3. [2] Equalization coefficient of basic public cultural services in Jiangxi Province in 2016.

\begin{tabular}{|c|c|c|c|c|c|c|c|c|c|}
\hline Area & $\mathrm{X1}$ & $\mathrm{X} 2$ & $\mathbf{X 3}$ & $\mathrm{X4}$ & $\mathbf{X 5}$ & X6 & $\mathbf{X 7}$ & X8 & X9 \\
\hline Nanchang & 78.94 & 0.01 & 0.017 & 0.01 & 0.187 & 97.67 & 98.93 & 5.789 & 0.006 \\
\hline Jingdezhen & 20.18 & 0.005 & 0.015 & 0.006 & 0.117 & 100 & 100 & 1.667 & 0.002 \\
\hline Pingxiang & 3.93 & 0.006 & 0.003 & 0.006 & 0.103 & 99.15 & 99.84 & 1.258 & 0.005 \\
\hline Jiujiang & 63.33 & 0.015 & 0.018 & 0.015 & 0.224 & 97.45 & 98.7 & 2.226 & 0.005 \\
\hline
\end{tabular}




\begin{tabular}{|c|c|c|c|c|c|c|c|c|c|}
\hline Area & $\mathbf{X 1}$ & $\mathbf{X} 2$ & $\mathbf{X 3}$ & $\mathrm{X4}$ & $\mathbf{X 5}$ & X6 & $\mathbf{X 7}$ & X8 & $\overline{X 9}$ \\
\hline Xinyu & 14.28 & 0.003 & 0.003 & 0.004 & 0.077 & 99.74 & 99.79 & 2.345 & 0.001 \\
\hline Yingtan & 4.02 & 0.004 & 0.005 & 0.004 & 0.0480 & 96.93 & 97.52 & 1.144 & 0.001 \\
\hline Ganzhou & 54.86 & 0.019 & 0.016 & 0.019 & 0.396 & 95.94 & 97.79 & 3.279 & 0.019 \\
\hline Ji'an & 52.86 & 0.015 & 0.014 & 0.015 & 0.254 & 98.26 & 99.59 & 1.722 & 0.012 \\
\hline Yichun & 39.78 & 0.010 & 0.012 & 0.011 & 0.147 & 99.15 & 98.38 & 3.513 & 0.017 \\
\hline Fuzhou & 32.76 & 0.012 & 0.010 & 0.014 & 0.152 & 98.82 & 99.86 & 3.216 & 0.002 \\
\hline Shangrao & 49.98 & 0.013 & 0.020 & 0.013 & 0.136 & 98.99 & 98.84 & 3.345 & 0.009 \\
\hline Average & 37.72 & 0.01 & 0.012 & 0.0106 & 0.167 & 98.37 & 99.02 & 2.68 & 0.007 \\
\hline standard deviation & 23.72 & 0.004 & 0.0057 & 0.0048 & 0.092 & 1.193 & 0.829 & 1.28 & 0.006 \\
\hline coefficient of variation & 0.623 & 0.409 & 0.471 & 0.436 & 0.552 & 0.012 & 0.008 & 0.478 & 0.842 \\
\hline Weight coefficient & 0.182 & 0.095 & 0.075 & 0.085 & 0.085 & 0.065 & 0.088 & 0.078 & 0.165 \\
\hline Equalization coefficient & 0.447 & & & & & & & & \\
\hline
\end{tabular}

As can be seen from Table 3, the average equalization coefficient in 2016 is greater than 0.4 , indicating that there is a large gap in basic public cultural services between cities in 2016, especially in terms of per capita cultural undertakings, the number of art performance groups owned by 10,000 people, and 10,000 The variability index of people in public library collections is between $0.5-0.6$, which shows that there is an imbalance in financial investment, talent team and cultural infrastructure construction between cities. Based on the equalization coefficients of various indicators of public cultural services in Jiangxi Province in 2016, the equalization coefficients of 2017 and 2018 are obtained in turn, and the three-year data from 2016 to 2018 are compared and analyzed.

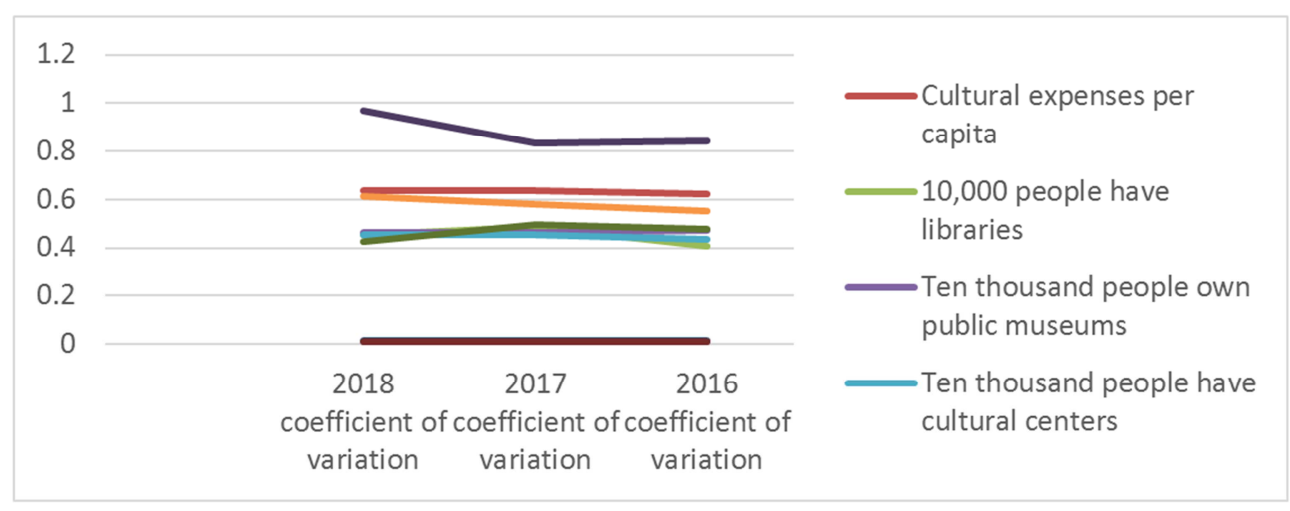

Figure 1. [3] The coefficient of variation of basic public cultural services in Jiangxi Province from 2016 to 2018.

It can be seen from Figure 1 that from 2016 to 2018, Jiangxi Province has the highest and stable equalization of the two indicators of comprehensive population coverage of radio and television, indicating that Jiangxi Province has reached equilibrium in terms of comprehensive population coverage of radio and television. The coefficients of variation of the three indicators of per capita cultural undertakings, museums owned by ten thousand people, and the number of cultural centers owned by ten thousand people are smaller each year from 2016 to 2018, indicating that their imbalances are narrowing. The coefficients of variation of the two indicators of 10,000 people owning libraries and 10,000 people owning cultural undertakings have increased from 2016 to 2017, but have decreased from 2017 to 2018, it shows that the gap in equalization of basic public cultural services has widened from 2016 to 2017, and the imbalance gap is narrowing from 2017 to 2018. The coefficient of variation of per capita cultural undertakings has increased from 2016 to 2017, and the coefficient of variation has decreased from 2017 to 2018, indicating that its equalization gap has decreased from 2016 to 2017, and from 2017 to 2018 The equalization gap is increasing. The coefficient of variation of this indicator for ten thousand people performing arts groups declined from 2016 to 2017, but the coefficient of variation increased from 2017 to
2018, indicating that the equalization gap was narrowing from 2016 to 2017. And the equalization gap between 2017 and 2018 has increased.

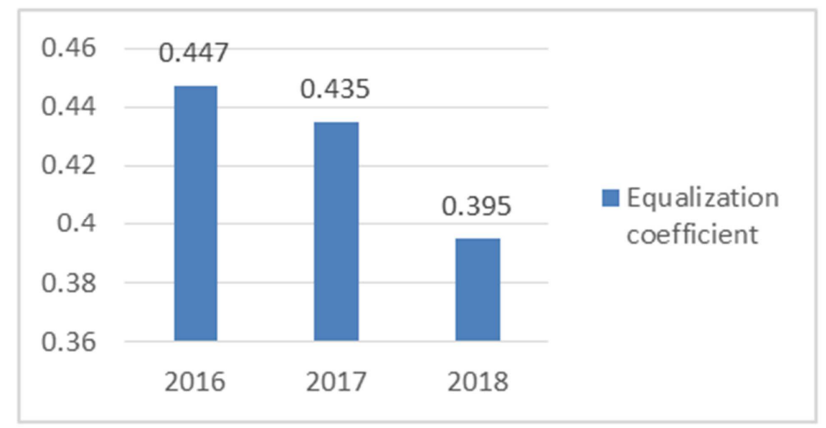

Figure 2. [4] Equalization coefficient of basic public cultural services in Jiangxi Province from 2016 to 2018.

Figure 2 shows that the equalization values of public cultural services in 2016, 2017, and 2018 in Jiangxi Province were $0.447,0.435$, and 0.395 , respectively, indicating that the gap between basic public cultural services in various cities in Jiangxi Province is continuously narrowing, but compared with developed regions, there are still Large gaps, cities in Jiangxi Province should increase financial investment and 
infrastructure construction when developing basic public cultural services, reducing the gap between cities.

\section{Problems in Basic Public Cultural Services in Jiangxi Province}

\subsection{The Gap Between the Equalization Levels Between Cities}

It can be seen from Table 2 that in terms of per capita cultural undertakings, the top ranking includes Nanchang 81.68 yuan, Jiujiang city 63.88 yuan, Ganzhou city 49.65 yuan, Shangrao city 39.23 yuan, the bottom ranking is Pingxiang city 8.82 yuan, Yingtan city 2.52 yuan, 16.38 yuan in Xinyu City and 24.82 yuan in Yichun City. The highest is Nanchang City, with a total of 81.68 yuan, and the lowest is Yingtan City, with a total of 2.52 yuan. The difference between the highest and the lowest value is 79.16 yuan, a huge difference. In terms of the number of libraries owned by 10,000 people, the top ranked regions are 0.019 in Ganzhou City, 0.015 in Ji'an City, 0.004 in Xinyu City, 0.004 in Yingtan City, 0.006 in Pingxiang City, and 0.006 in Jingdezhen City. The number of libraries varies greatly, and the difference between the highest value and the lowest value is 0.015 . The same is true for the number of museums and cultural centers owned by 10,000 people. In terms of library books owned by 10,000 people, the areas with higher values include 0.446 volumes in Ganzhou City, 0.226 volumes in Nanchang City, and 0.351 volumes in Jiujiang City, while the areas with lower values are 0.05 volumes in Yingtan City, 0.086 volumes in Xinyu City, and 0.103 volumes in Pingxiang City. 10,000 people have Ganzhou City with the highest library collection, with a value of 0.446 , the lowest is Yingtan, with a value of 0.050 , and the difference between the highest value and the lowest value is 0.396 . It can also be seen that the degree of equalization of radio and television coverage is high, and the difference between regions is small. Each city has a relatively low degree of equalization in the employment of cultural undertakings, which is manifested as 5.321 people in Nanchang City, 3.758 people in Ganzhou City, 3.265 people in Xinyu City, but only 1.51 people in Pingxiang City, 1.327 people in Jiujiang City, and 1.569 people in Jingdezhen City, Regional differences are obvious. In terms of ten thousand people performing arts groups, 0.086 in Fuzhou, 0.073 in Shangrao, 0.08 in Nanchang, 0.001 in Xinyu, 0.002 in Jingdezhen, and 0.013 in Yingtan. Through the comparison of the above values, it can be concluded that the basic public cultural services of various cities in Jiangxi Province have large regional differences in various indicators. Looking at it horizontally, in addition to the coverage of radio and television, the coefficient of variation of each index is large, indicating that the level of equalization in each region is low. Vertically, Nanchang City, Ganzhou City, Jiujiang City, Jingdezhen City and other cities have higher numerical values, while Pingxiang City, Yingtan City, Xinyu City, and Yichun City have lower numerical values. The basic public cultural service resources of Jiangxi Province are mainly concentrated in areas with better economic development, which are manifested in the improvement of basic public cultural hardware facilities, sufficient basic public cultural service personnel, strong awareness of public cultural service, and the increasing penetration of public cultural service activities, People in the community can enjoy unique artistic performances in their daily lives. In areas with poor economic development, limited by natural conditions and economic development levels, public cultural infrastructure is not perfect, human resources and construction funds are insufficient, and the number of public participation in public cultural service activities is limited. There is a large regional gap in the equalization of basic public cultural services in Jiangxi Province, and it is necessary to strengthen the construction of basic public cultural services in economically backward regions.

\subsection{Unequal Financial Input}

Fiscal input refers to the expenditure of the public finance on social public service management, social security and other aspects of social public utilities. Fiscal investment plays a key role in the process of equalizing public services, it is the basis and guarantee for equalizing public services. The amount of financial investment largely determines the quantity and quality of public service construction. It can be seen from Table 2 that in 2018, Jiangxi Province had a large gap in per capita cultural undertakings in 2018, Nanchang City was far ahead with 81.68 yuan, Jiujiang City invested 63.88 yuan, and the remaining 9 cities were all below 50 yuan, The per capita cultural undertaking cost of Yingtan City is less than 20 yuan, the gap between cities is large, and the per capita cultural undertaking cost of most cities is low. The coefficient of variation in the past three years shows that the coefficient of variation of per capita cultural undertakings in all cities in Jiangxi Province is above 0.5 , indicating a huge difference. In addition, as a whole, the per capita cultural undertaking fee of Jiangxi Province is lower than the national level, and it can be seen that the investment of cultural finance in Jiangxi Province is uneven. As a provincial capital city, Nanchang has sufficient investment in cultural undertakings to provide advanced public culture for the public in the region The service facilities, rich and colorful cultural activities and art performance groups, and sufficient public cultural service professional talent teams have laid a solid material foundation for the realization of equalization of basic public cultural services. In other economically backward regions, the financial investment for public cultural services is relatively small, and it is difficult to meet the needs of service facilities and talents. It is necessary to strengthen the financial investment of cultural undertakings in economically backward regions and promote the equalization of public cultural service financial investment.

\subsection{Insufficient Talent team Building}

In the process of basic public cultural service construction, specialized public cultural service talents are needed to formulate policies, guidelines, determine the allocation and adjustment of public cultural service resources, and be 
responsible for the promotion of relevant policies and the provision of public cultural services. Therefore, a large number of professional service personnel run through the entire process of public cultural service construction. The lack of service talents will make the construction of public cultural services lack motivation and vitality, and service activities cannot be carried out normally. From Table 2, it can be seen that the number of employees with cultural undertakings in different cities varies greatly and is unevenly distributed. From 2016 to 2018, the coefficients of variation of employees with cultural undertakings in various cities were all above 0.5 , indicating that the equalization of basic public cultural services in Jiangxi Province has inequalities in the indicators of employees. In addition, Jiangxi Province still has shortcomings in the training of talents, and the problem of aging staff is more serious, lacking vitality and creativity, the establishment of grass-roots cultural units is low, which makes it impossible for talents with specialized skills to enter. Many regions can only introduce talents by means of temporary recruitment or project management, but this results in different pay for equal work or even low pay for high-tech workers. Unfair treatment makes it difficult to attract talent. At the same time, the staff of cultural centers and museums are basically one person, one station responsible for the system and take on multiple roles, making it difficult to fully engage in public cultural services. The basic public cultural service staff in Jiangxi Province are not of high professional quality, their service concept is not in place, they cannot keep up with the needs of the construction of public cultural services, they cannot adapt to the changing network information era, and they lack professional public cultural management talents and scientific researchers. The construction of talents for public cultural services lacks an incentive mechanism. Generally, the salaries of public cultural service workers are relatively low and the preparation of plans is low. Due to lack of development opportunities in economically underdeveloped areas, it is difficult to retain talent.

\subsection{Infrastructure Construction Needs to be Improved}

Basic public cultural service infrastructure mainly includes libraries, museums, cultural art galleries, cultural stations, radio and television projects, etc. Having a complete public cultural service infrastructure is the basis for promoting the equalization of basic public cultural services. All cities in Jiangxi Province need to increase capital investment in infrastructure and accelerate the implementation of a fully covered public cultural service foundation. In 2018, the coefficient of variation of basic public cultural services in various cities in Jiangxi Province can be concluded that the coefficient of variation in libraries, library collection per capita, and construction of cultural centers is relatively high, indicating that the degree of equalization in each city is low. The cities with higher numerical values have more developed economies and more comprehensive infrastructure construction. Specifically, Nanchang, Jiujiang, Ganzhou and Jingdezhen have more cultural infrastructure, while Pingxiang, Xinyu, yingtan and Yichun have less cultural infrastructure.
The construction of public cultural service infrastructure is mainly concentrated in economically developed areas, rich in resources, and a large population. The quality and quantity of the construction of libraries, museums, and cultural art galleries are relatively high. The comprehensive coverage of radio and television can basically reach $100 \%$ coverage, and can provide the public with the basic public cultural service facilities needed. The economically backward regions are developing slowly, and the infrastructure construction in all aspects is relatively simple, which cannot meet the needs of the public. Moreover, there is the phenomenon of unreasonable construction. Building cultural infrastructure in places where the population is not concentrated has led to many cultural facilities being shelved for a long time after being built, and the phenomenon of waste is serious. At the same time, the lack of management personnel of public cultural infrastructure has resulted in the long-term maintenance of its facilities and the sparse number of visitors. At the same time, the construction of basic public cultural facilities does not match the needs of the public, and there is a lack of understanding of public needs. Therefore, it is necessary to strengthen and improve the construction of public cultural service infrastructure.

\section{Countermeasures to Promote the Development of Basic Public Cultural Services in Jiangxi Province}

Through the above empirical analysis, this article can draw the following conclusions: According to the division of the three regions of southwest, north and middle of Jiangxi, the development level of basic public cultural services in northern Jiangxi (Nanchang, Jiujiang, Jingdezhen) is higher than that of Ganzhong (Jian, Fuzhou) City) area, and the development level of basic public cultural services in Gannan (mainly Ganzhou) area is higher than that in Ganzhong area, which has a strong correlation with the economic development level of cities in the northern Jiangxi area; The secondary indicators of public cultural services exhibit unbalanced characteristics; the cities with a relatively high level of economic development may not have equal coefficients of variation in basic public cultural services; the degree of inequality in basic public cultural services among 11 cities in Jiangxi Province This has been improved, and the inequality of basic public cultural services in the central cities of Jiangxi is more obvious. Based on the above research, the following countermeasures and suggestions are proposed:

\subsection{Promote Regional Coordinated Development and Establish a Service Model for Coordinated Development of Urban and Rural Areas}

Regional coordinated development is one of the "five co-ordinations" proposed by the Third Plenary Session of the Sixteenth Central Committee of the Communist Party of China. It aims to gradually reverse the widening regional development gap by improving market mechanisms, 
cooperation mechanisms, and mutual assistance mechanisms. The regional economic development in Jiangxi Province is obviously differentiated and has an expanding trend, but in recent years, the expansion rate has slowed down, and the economy has developed in a ladder-like manner. When promoting the coordinated development of regional economy, Jiangxi Province should focus on strengthening investment in peripheral areas and narrowing the gap between economically backward areas and developed areas. First, when formulating development plans, we should fully consider the differences in economic development of various regions, take advantage of our own circumstances, ensure efficiency and take into account fairness, and actively support, encourage, and guide economically backward regions to strive for their own development. The second is to cultivate and develop peripheral economic belts, focusing on cultivating the economic belts of Nanchang, Ganzhou, Jiujiang, and Jingdezhen, and promoting the economic pattern of comprehensive development with a point. At present, to achieve the goal of equalization of basic public cultural services in Jiangxi Province requires full attention to the development of rural public cultural services. In the process of improving the equalization of public cultural services, governments at all levels should focus on rural areas, increase investment in the construction of rural public cultural services, give full play to the supporting role of township cultural centers and libraries, and organize and guide township comprehensive cultural services Station, determine the main functions of county-level cultural centers and libraries in radiating and driving regional public cultural services, and increase the strength of township cultural centers, including funding, staffing, and management model research, and strive to complement the impact of equalization construction Existing "short board".

\subsection{Establish a Multiple Investment Mechanism Based on Public Financial Investment}

The source of funds for the equalization of basic public cultural services in Jiangxi Province needs to shift from a single government input to a multi-input mechanism that focuses on government input, with multiple inputs from individuals, third-party organizations, and enterprises. Achieving equality in basic public cultural services is an important manifestation of the public nature of public finance. As the main body of public cultural service investment, it is essential for the government to establish public finance. It will be improved from three aspects. One is to strengthen the stimulation of the social donation incentive mechanism and establish a public cultural development fund. First, it will attract investors and sponsors, companies, groups and individuals to subsidize public cultural services through policies such as tax reduction, tax exemption, financial support, commendation titles and project support. The second is to concentrate social forces to participate in the construction of public cultural services. Encourage and support individuals, enterprises, and society to voluntarily contribute to the construction of social public cultural activity venues or public cultural service hardware facilities, and establish public cultural service construction funds under the leadership of government public financial funds. The third is to improve the fiscal transfer payment equalization system. Government fiscal transfer payment refers to the purpose of bridging the fiscal gap in regions with weak fiscal strength, narrowing the financial gap between regions, and promoting the equalization of basic public services between regions. Its fiscal funds are mainly recovered by collecting taxes. The perfection of the fiscal transfer payment equalization system is mainly to promote the horizontal and vertical balance of fiscal funds between localities and to achieve the equalization of various basic public service resources between regions.

\subsection{Strengthen the Construction of Talents for Basic Public Cultural Services}

Cultivating human resources for public cultural services is crucial, because from the formulation, implementation, implementation and supervision of public cultural service policies, professional public cultural service personnel are needed. Without professional public cultural service personnel, it is difficult to achieve equalization of basic public cultural services in all cities. At present, the basic construction of basic public cultural service personnel in Jiangxi Province is mainly concentrated in Nanchang, Ganzhou, Jiujiang and other regions, while the lack of professionals in Pingxiang, Xinyu, Yingtan and other public cultural services, the lack of professional service personnel in these areas, restricts the basic public culture The improvement of service level also makes the construction of cultural services in the region lack vitality and creativity. In the process of achieving equalization of basic public cultural services, we must attach importance to the cultivation of professional cultural management talents and cultural and artistic performance talents, and strengthen the construction of basic public cultural service talents. In the process of equalization of basic public cultural services in Jiangxi Province, professional staff are needed, mainly including the establishment of incentive mechanisms to attract talents, increase the establishment and treatment of public cultural service personnel according to actual needs, strengthen the training of professional talent teams, and optimize the construction of professional talent teams Structure, improve the quality of public cultural service talents, strengthen the principle of openness, equality and merit-based recruitment, and establish a team of high-quality talents who will provide quality services to the public wholeheartedly. In addition, to strengthen the cultivation of rural public cultural service talents, provincial governments need to increase support for rural public cultural services, which can not only ease urban resource constraints but also promote the construction of rural public cultural service infrastructure.

\subsection{Strengthen Basic Public Cultural Service Infrastructure Construction}

Basic public cultural services are based on corresponding 
cultural facilities, and public cultural services and cultural activities need to be provided to the public with the help of cultural infrastructure. Equal public cultural service infrastructure configuration is essential. The current situation of basic public cultural service infrastructure allocation in Jiangxi Province is that resources are mainly concentrated in Nanchang and Ganzhou, especially in Nanchang. Nanchang's economic development level is higher than other cities, with advanced cultural facilities, cultural equipment, libraries, and museums. The construction of the cultural center is complete, the exhibits are rich, and cultural facilities such as cultural stations and cultural parks are constantly developing. The economic development level of Jiujiang is relatively fast, and the construction of basic public cultural service facilities has been continuously completed. Although it is higher than the development level of Xinyu and Yingtan, it still has a big gap with the basic public cultural service infrastructure configuration level of Nanchang. However, the construction of basic public cultural service facilities in Xinyu City, Pingxiang City, Yingtan City and other places is backward, outdated and slow to update, and the diversity of basic public cultural services is difficult to meet the needs of the public. At present, Jiangxi Province still has unequal phenomena in the construction of museums, libraries and cultural centers. Facilities such as libraries, museums, and cultural centers are unevenly distributed, mainly concentrated in the main urban area, and a few remote areas even lack public cultural service facilities or outdated facilities. Therefore, governments at all levels in Jiangxi Province should strengthen the construction and improvement of public cultural service facilities in remote areas, especially the supplement and improvement of basic public cultural service facilities in economically backward areas, and increase the construction of related service facilities. This is the most direct and most important way for the public to enjoy basic public cultural services, and it is conducive to meeting their basic cultural needs and achieving the goal of equalization at an early date.

\section{Acknowledgements}

This paper is supported by the Jiangxi Province University Humanities and Social Sciences Research 2019 Project: "Promoting Equalization of Public Cultural Services in Urban and Rural Areas in Jiangxi Province" (No. GJ19112).

\section{References}

[1] Jiangxi Statistics Bureau. Jiangxi Statistical Yearbook [J]. Beijing: China Statistics Press, 2017-2019.

[2] Zou Jinhui, Zhang Yaqi, Zhou Yaqian. A review of the major projects of the National Social Science Fund "Promoting the Research on Standardization and Equalization of Basic Public Cultural Services in my country" $[\mathrm{J}]$. Library Work and Research, 2020 (03): 5-12
[3] Yang Lin, Yang Guangyong. Evaluation of the quality of basic public cultural service supply and its improvement Practice from Shandong Province $[\mathrm{J}]$. Shandong Social Sciences, 2020 (02): 105-111.

[4] Yu Aiguo, Zhang Weifeng. A study on the regional gap in the development level of basic public cultural services in my country [J]. Hundred Arts, 2018, 34 (02): 113-118+152.

[5] Jiang Mimu, Zhang Jing. Measurement of the efficiency of fiscal expenditure on basic public cultural services in China [J]. Jianghan Forum, 2017 (03): 102-107.

[6] Ke Ping, Zou Jinhui, Li Mengling, Hu Yinxia. Research on the Reasonable Value Orientation of Equalization of Basic Public Cultural Services [J]. Journal of National Library of China, 2017, 26 (05): 3-9.

[7] Fu Caiwu, Zhang Weifeng. Research on the Equalization of Basic Public Cultural Services-Model Construction and Empirical Analysis [J]. Library Journal, 2018, 37 (08): 4-13.

[8] Wang Luozhong, Li Fan. Basic public cultural services in my country: Index system construction and regional gap measurement $[\mathrm{J}]$. Economic and Social System Comparison, 2013. (01): 184-195.

[9] The General Office of the State Council. The 13th Five-Year Plan for Equalization of Basic Public Services [Z]. 2017.01.23.

[10] Jiangxi People's Government. The 13th Five-Year Plan for Jiangxi National Economic and Social Development [Z]. 2016.10.19.

[11] Luo Xianfang. Research on the supply mode of public cultural services [J]. Information Exploration, 2018 (06): 32-35.

[12] Zhang Linna, Zhu Yelai. Commentary on the status quo of public cultural service research at home and abroad and its future prospects $[\mathrm{J}]$. Journal of Xi'an University of Finance and Economics, 2013 (3): 123-128.

[13] Differences and spatial patterns of basic public cultural services in my country [J]. Shi Tao, Hu Tao, Yan Yuexia, Zhang Yanfeng. Knowledge Management Forum. 2014 (06).

[14] Li Min. Analysis on the dynamic supply characteristics and equalization path of basic public cultural services in Jiangsu [J]. Journal of Southeast University (Philosophy and Social Sciences Edition), 2017 (05): 67-73+147.

[15] Hao Guoqing. New problems and mechanism innovation in the process of equalization of basic public services [J]. Journal of Hubei Administration College. 2015 (5): 49-53.

[16] Ren Sufang. Suggestions for promoting the equalization of basic public cultural services [J]. Shanxi Finance and Taxation, 2012 (06): 22-23.

[17] Shi Tao, Hu Tao, et al. Differences and spatial patterns of basic public cultural services in my country $[\mathrm{J}]$. Knowledge Management Forum, 2014 (06): 1-7.

[18] Yang Yiqing. The path of urbanization from the perspective of equalization of public services [J]. Modern Economic Research, 2016 (5): 15-19.

[19] Peng Leiting, Pi Yanfang. An empirical study on the equalization of basic public cultural services in Jiangsu Province [J]. Library Journal, 2018, 37 (09): 27-36+110. 